\section{Contingency management treatments}

\author{
NANCY M. PETRY
}

\section{Summary Contingency management is highly efficacious in improving outcomes in substance misuse. Whereas a great deal of research has evaluated these interventions empirically, few treatment providers integrate this approach in practice. The rationale for contingency management is described, with a call for expansion of this technique outside the USA.}

\section{Declaration of interest None.}

N.P. receives research funding from the National Institute of Health.

Contingency management treatments are widely used in drug misuse research and are slowly gaining popularity in clinical settings in the USA. These are interventions in which substance misusing people receive tangible, positive reinforcers for objective evidence of behaviour change. For example, patients receive a voucher, exchangeable for retail goods and services, whenever they submit a urine specimen that tests drug negative. A clinic-managed 'bank account' is established, in which the first negative sample results in US\$2.50, the second US $\$ 3.75$, the third US\$5.00 and so on. Once people have earned enough vouchers for an item of their choosing, they request their vouchers be exchanged for a television, stereo equipment, clothing, cinema tickets, etc. Staff purchase requested items, so cash is not handed directly to patients, and certain items (e.g. weapons, liquor) are not approved for voucher spending.

Studies evaluating the efficacy of such interventions have more or less ubiquitously demonstrated positive effects when compared with more traditional forms of substance misuse treatment (Petry, 2000; Lussier et al, 2006). In the classic voucher study by Higgins et al (1994), cocaine-dependent individuals who received vouchers achieved an average of
11.7 weeks of continuous cocaine abstinence $v .6 .0$ weeks among those who received the same psychotherapy without the voucher component. Subsequent studies isolated the benefits of vouchers to their contingent nature, rather than mere availability. Higgins et al (2000) randomly assigned 70 cocaine-dependent outpatients to a contingency management condition in which receipt of vouchers was dependent upon submission of cocaine-negative samples or a yoked control condition in which vouchers were provided independent of urinanalysis results. Almost $40 \%$ in the vouchercontingent condition achieved 12 or more weeks of continuous abstinence compared with little more than $10 \%$ in the yoked condition.

Although clearly efficacious in enhancing durations of drug abstinence in substance misusing patients, contingency management procedures have not yet been widely adapted by community-based treatment programmes. One barrier relates to their costs. The magnitude of vouchers available is directly associated with efficacy. Usually, patients can earn up to about US\$1200 in vouchers over a 12-week intervention, and studies that provide lower voucher amounts demonstrate less positive effects (Petry, 2000).

Over the past 7 years, we have been developing and testing a modified contingency management approach that appears to lower costs while retaining efficacy. Rather than earning a voucher worth a set monetary amount, patients earn the opportunity to draw a slip of paper from a fishbowl. Each draw is associated with a chance to win a prize. Prizes range in value from US\$1 items (bus tokens, food or toiletries, socks, make-up), US\$20 items (sweatshirts, watches, handheld CD players, dish sets), to US\$100 items (television, DVD player, stereo equipment). About half the slips are not associated with a prize and simply state: 'Good job!' Winning slips vary inversely in proportion to the value of the prizes, such that only one slip is the US $\$ 100$ prize, whereas about $7 \%$ to $8 \%$ are the moderate-priced prizes and $42 \%$ are small prizes.

The prize system has features similar to those of the voucher system and behavioural therapy in general. That is, the number of draws earned increases with successive abstinence, prizes are individualised with many items available, and the exchange delay between behaviour and reinforcer is minimised. However, overall magnitudes of expected winnings are arranged to be about one-third of those of the voucher system. Most participants in prize contingency management studies earn between US\$80 and US\$200 during a 12-week treatment period.

Prize contingency management is efficacious in improving outcomes of many substance misusing populations. Thus far, it has shown beneficial effects in alcohol (Petry et al, 2000), cocaine (Petry et al, 2005a), and cocaine-opioid misuse (Petry $\&$ Martin, 2002). Petry and colleagues (2005a) found that the prize system was as efficacious as the voucher system. Furthermore, a recent multicentre study of the prize system was conducted in drug-free (non-methadone) and methadone clinics throughout the USA. Over 800 stimulant misusers were randomised to either treatment as usual or treatment as usual plus prize contingency management for 12 weeks. In the non-methadone settings, contingency management significantly improved retention in treatment and longest durations of stimulant abstinence achieved (Petry et al, 2005b). In methadone clinics in which retention rates are usually high because of the reinforcing properties of methadone itself, no differences in attrition were noted between the groups, but proportions of negative samples submitted were significantly higher in the prize system (Peirce et al, 2006). These data suggest widespread applicability of contingency management procedures and their utility as an adjunct to any other form of therapy provided.

The development of contingency management in the USA is in part a reflection of that country's society. The US National Institute on Drug Abuse supports about $85 \%$ of all drug misuse research throughout the world, thereby allowing for discovery and testing of novel therapies. Contingency management, in particular, stems from the behavioural 
therapy movement, with strong roots in North American academic and applied psychology. One could also consider contingency management a reflection of a capitalistic society with its emphasis on incentives and tangible items.

Although North American culture led to the development of contingency management therapy, it is also responsible for the relatively poor adoption of this efficacious intervention into practice. In the USA the most widespread treatment for substance misuse is 12-step (Alcoholics Anonymous) in nature. The stance toward treatment is usually abstinence oriented, with confrontational approaches not uncommon. Harm reduction philosophies are often met with resistance, and some of the states in the USA still ban methadone treatment. Consequently, contingency management is the least widely recognised or integrated treatment approach in practice (McGovern et al, 2004), despite strong evidence of efficacy.

In the UK and Europe, policies related to pharmacological maintenance and harm reduction can be more liberal in nature. Thus, it is somewhat surprising that contingency management interventions have yet to be introduced outside the USA. Although funding for drug misuse treatment is low throughout the world and full-scale costeffectiveness evaluations of contingency management have yet to be conducted, one could argue that the costs of even a single emergency case or drug-related crime exceed the resources required for prizebased contingency management.

Some ethical issues have also plagued contingency management. These include concern that patients may exchange prizes or voucher earnings for drugs, and that the prize-based technique may mimic gambling. North American studies have

NANCY M. PETRY, PhD, University of Connecticut School of Medicine, 263 Farmington Avenue, Farmington, CT 06030-3944, USA. Tel: + 860679 2593; fax: + 860679 1312; email: petry@psychiatry.uchc.edu

(First received 23 January 2006, final revision 16 April 2006, accepted 2 May 2006)

found that payments to drug users rarely induce drug use (Festinger et al, 2005), and a drug-positive sample would reset vouchers or draws available in contingency management programmes. Petry et al (2006) found no increase in gambling among large samples of substance misusers participating in prize contingency management studies. Moreover, the likeness to gambling is only superficial, as nothing of value is risked in contingency management, whereas risk is the defining feature of gambling.

Politics and ideology will clearly impact on the expansion of this technique in practice. However, objections toward 'paying drug abusers to do what they should do anyway' may be somewhat tempered by pragmatic and Machiavellian principles of doing whatever works best for this difficult-to-treat and often disenfranchised population. Evaluation of contingency management approaches in other cultures and societies ultimately may enhance prevention efforts in high-risk groups and improve treatment outcomes of drug misusers throughout the world.

\section{REFERENCES}

Festinger, D. S., Marlowe, D. B., Croft, J. R., et al (2005) Do research payments precipitate drug use or coerce participation? Drug and Alcohol Dependence, 78, 275-281.

Higgins, S. T., Budney, A. J., Bickel, W. K., et al (1994) Incentives improve outcome in outpatient behavioral treatment of cocaine dependence. Archives of General Psychiatry, 5I, 568-576.
Higgins, S. T., Wong, C. J., Badger, G. J., et al (2000) Contingent reinforcement increases cocaine abstinence during outpatient treatment and I year of follow-up. Journal of Consulting and Clinical Psychology, 68, 64-72.

Lussier, J. P., Heil, S. H., Mongeon, J. A., et al (2006) A meta-analysis of voucher-based reinforcement therapy for substance use disorders. Addiction, 10I 192-203.

McGovern, M. P., Fox, T. S. \& Xie, H. (2004) A survey of clinical practices and readiness to adopt evidencebased practices: dissemination research in an addiction treatment system. Journal of Substance Abuse Treatment, 26, 305-312.

Peirce, J. M., Petry, N. M., Stitzer, M. L., et al (2006) Lower-cost incentives increase stimulant abstinence in methadone maintenance community treatment: results of the National Drug Abuse Treatment Clinical Trials Network Multi-site Study. Archives of General Psychiatry, 63, $201-208$

Petry, N. M. (2000) A comprehensive guide for the application of contingency management procedures in standard clinic settings. Drug and Alcohol Dependence, $\mathbf{5 8}, 9-25$.

Petry, N. M., Martin, B., Cooney, J. L., et al (2000) Give them prizes and they will come: contingency management for the treatment of alcohol dependence. Journal of Consulting and Clinical Psychology, 68, 250-257.

Petry, N. M. \& Martin, B. (2002) Lower-cost contingency management for treating cocaine-abusing methadone patients. Journal of Consulting and Clinical Psychology, 70, 398-405.

Petry, N. M., Alessi, S. M., Tedford, J., et al (2005a) Vouchers versus prizes: contingency management for treatment of substance abusers in community settings. Journal of Consulting and Clinical Psychology, 73. |005-III4.

Petry, N. M., Peirce, J. M., Stitzer, M. I., et al (2005b) Effect of prize-based incentives on outcomes in stimulant abusers in outpatient psychosocial treatment programs: a national drug abuse treatment clinical trials network study. Archives of General Psychiatry, 62, II48-II56.

Petry, N. M., Kolodner, K. B., Li, R., et al (2006) Prize-based contingency management does not increase gambling. Drug and Alcohol Dependence, in press. 